\title{
Storytelling in a First-Year Seminar
}

\author{
Donna M. Ehrlich, $\mathrm{PhD}$ \\ Associate Professor, Computer Information Systems \\ Park University \\ Jeff A. Ehrlich, EdD \\ Associate Professor, College of Management \\ Park University \\ April Haberyan, PhD \\ Nurse Residency Program Manager \\ Truman Medical Center
}

\begin{abstract}
Historically, storytelling has been a way to pass knowledge between generations and to facilitate an understanding of beliefs. The aim of this current research was to explore the value of storytelling in the higher education classroom, to address the question "how can sharing stories assist students in constructing new knowledge in the classroom?" The purpose of the study was to see if teaching students how to tell a story and having them tell a story in a Freshman seminar class would enhance and enrich the quality of the knowledge they gained in discussions as students. The research utilized the lens of constructivism and a community of inquiry. Findings shed light on the perceptions of the students and the level of classroom engagement after experiencing guided storytelling. The results demonstrated an increase in engagement in the classroom. Students did not feel they needed story telling guidance, but they did feel storytelling was valuable to their overall experience.
\end{abstract}

Storytelling is an ancient art used in many cultures both for entertainment and for education across generations and disciplines. Historically storytelling was used to explain complex interactions as the only means to share information about life and life's lessons between generations. In today's academic world, storytelling is gaining renewed attention from practitioners and academics across a variety of disciplines, as it expands into the formal pedagogy of education. Researchers are expanding specific theories and knowledge about the practice of storytelling as it applies to the formal classroom and education (Hamstra, 2017). Learning is a complex process, involving a number of different levels; using storytelling, the different levels are combined into a reflective technique with a human side becoming "both a student-support strategy and a learning activity.... and can be used as a cognitive construct" (van Rensburg et al., 2018, p. 608). Applying this complexity, storytelling in the classroom fosters critical thinking, communication, collaboration, and creativity (Cherry, 2017). When a student listens to a story, not only are the vocabulary areas of the brain activated but also the sensory areas of the cortex as the topic is given life through a story (Chow et al., 2014). According to Mar and Oatley (2008) the brain experiences the story in the same way it would as if the events were actually happening to the listener. In addition, stories foster empathy and allow us to understand the thoughts and feelings of others (Mar \& Oatley, 2008; Zak, 2015). As a result, storytelling can create interconnectedness and a sense of community in the classroom.

InSight: A Journal of Scholarly Teaching 
Storytelling has been used in multiple formats in disciplines across higher education. Health care, especially nursing, has been using storytelling to enhance the learning opportunities of students as they develop their clinical skills (Keoning \& Zorn, 2002). The use of storytelling in classrooms continues to grow, and research demonstrates this method works as an effective tool, provided the student is engaged and understands how to tell a story (Fahy, 2007). Previous research primarily focused on the instructor telling the

...storytelling can create interconnectedness and a sense of community in the classroom. story or a student with limited training in storytelling telling a story. This research focused on the impact of students learning how to tell stories. What difference does it make if a student learns how to tell a story, and what is the perception of the student in the storytelling assignment? In this research, a student is assigned to tell a story about an activity they engaged in on campus rather than just telling the facts about the activity. The student is then asked to relate this activity to the foundational literacies of the University. This activity encourages the student to deepen their understanding of the University liberal arts educational culture and its purpose in education.

In order to study the differences experienced in this assignment, half of the students were given a guide on how to tell a story. The other half were simply assigned to tell a story. The students were then asked to provide feedback through focus groups and survey monkey. The feedback requested was to ask if they felt having or not having storytelling guidance was helpful in telling a story. They were also asked if telling a story was beneficial both to hear other student's stories and to see if their story telling was enriching to their understanding of the literacies. In order to gain access to the student's information an IRB (Appendix A) was submitted and approved providing the guidelines to allow the students to participate.

Community of inquiry and constructivism of knowledge were combined to create the framework for the study. Community of inquiry provided the different levels as the assignment required a social and cognitive approach inside the learning community. This approach, along with the distance presence of stepping back to view the constructs, provided a lens to create the story, which is then used to develop or construct new knowledge that can be applied professionally (Phillips et al., 2017).

\section{Community of Inquiry}

The community of inquiry framework assumes learning occurs in a community of individuals with a common goal of constructing knowledge through critical reflection. Community of Inquiry model suggests that certain collaborative interactions create a "distance presence", which facilitates the emergence of a new educational experience (Garrison \& Vaughan, 2008). This experience creates an ability to go beyond the previous understanding to a new level of being (Garrison, 2009; Garrison et al., 2010).

Previous research posits that the three presences (social, cognitive and teaching) found in a community are interconnected. Social presence is the interaction students would have with other students through the assignment, which is to attend an event on campus, thus creating a need to be socially present. The cognitive approach 
is personal mental awareness, creating a deeply personal connection. Teaching is a presence of understanding a student's experience in a classroom setting (Sterman, 1991).

Each presence exhibits a unique level of being. These three presences may also have an influence on one another. The interconnectedness between the three presences underpins the concept that teaching presence has a regulatory role while social is the application that comes through behavior from the individual level of current understanding. (Garrison \& Vaughan, 2008).

In our research, students are required to complete a social assignment and use a cognitive approach in telling a story. This allows a "distance presence" to the activity linked to the literacies. The distance presence provides a level for a student to reflect upon the experience and through this lens begin to construct a new level of understanding (Phillips et al., 2017).

In a community of inquiry, Sterman's (1991) requirements of a good model states that assessment should be made based upon utility for purpose rather than determining or grading a student response to an actual activity. For this study, the storytelling exercise was intended to create a convergence of learning through an online environment involving the students interacting socially through their own cognition. The story creates a distance from just the facts and provides applications and shared experiences. Together the students interact, sharing their own lived experience through a story, and through the distance presence developed by the story from the specific assigned memorization of facts. The goal was to build a sense of inquiry through storytelling about their lived experiences, thus creating an educational experience. As the story is told by the student about their personal experience, the construction of new knowledge is formed. This leads to the next lens for this research: utilizing student constructivism.

Figure 1

Community of Inquiry Model (Garrison et al., 2010)

\section{Community of Inquiry}

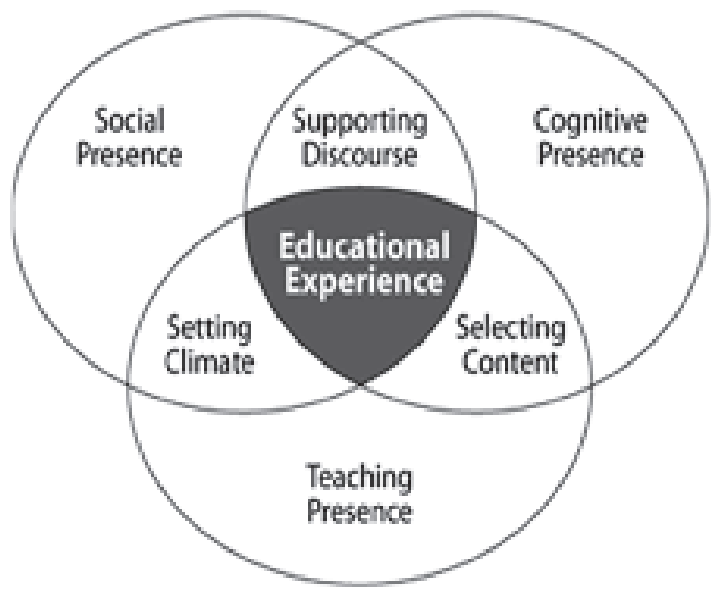

Communication Medium

InSight: A Journal of Scholarly Teaching 


\section{Student Constructivism}

Constructivist learning dates back to the 1930s as an educational philosophy (Pass, 2007). In an elementary sense, the constructivist model of learning assumes learning as a process of constructing or making something, usually through some sort of conceptual modeling. The premise of the model is that people learn by making sense out of the learning experience through encounters. Thus, curriculum that fosters the learning process requires mental engagement, not passive attendance in a typical student/teacher relationship. In essence, the student becomes an interpreter of the curricular content and incorporates the views of peer students into the construction of new knowledge. By creating a place where learners work together and support each other as they use a variety of tools (including storytelling), the researcher supports achievement of their learning goals.

As educators we have control over what is taught, but less control over what students learn (Brooks \& Brooks, 1999). At the same time, as the curriculum is designed to ensure that students construct their own meaning of the content, it is also designed to ensure that students learn the same concepts at the same time. Each student still constructs their own meaning through their own construction of knowledge.

In his early work Jean Piaget looked at children creating a transformative experience of constructing new knowledge, while learning through individual work on an assignment or activity. Similarly, Lev Vygotsky observed the learning process of constructivism, except he observed social interactions, with learning coming through those interactions with other students (Pass, 2007).

Over time, these two combined their ideas and developed a deeper understanding of the way in which students construct knowledge, combining the social and cognitive approaches (Pass, 2007). Vygotsky was focused on social constructivism and Piaget was cognitive, emphasizing the individual and how each constructs knowledge through learning behaviors. Vygotsky viewed learning and application in the classroom through social interaction approaches, interacting with the environment as a primary driver.

Figure 2

Student-centered Education and Constructivism (Krahenbuhl, 2016)

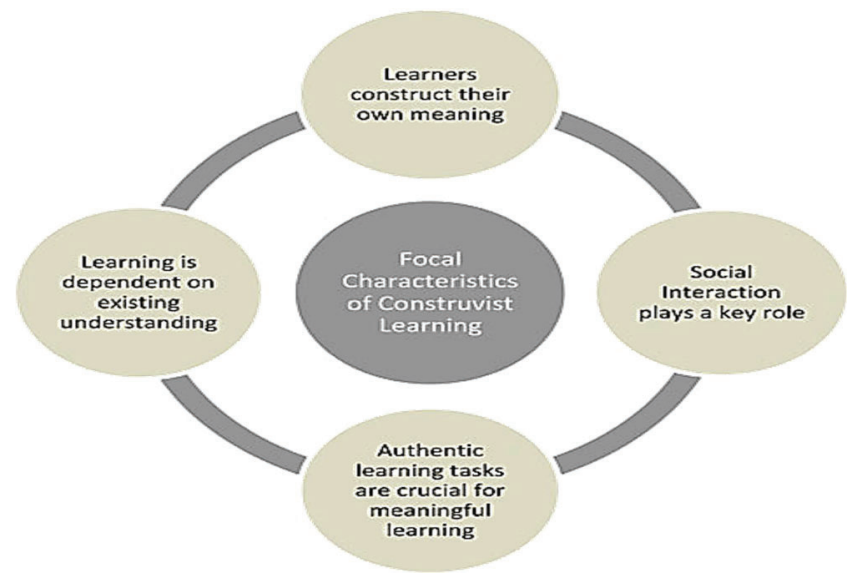


In the 1990s, constructivist theory increased its importance in the learning environment, combining the cognitive and social aspects of learning into teaching practice (Dunlap \& Grabinger, 1996). Powell and Kalina (2009) state,

There are different perspectives on learning and what constitutes an effective constructivist classroom. However, acquiring knowledge, experience or understanding is common to all of them. How to create an effective constructivist classroom and measure the successful results of students' learning, would include alternate teaching practices. (p. 248)

Thus, for the purpose of this research the Constructivist model is combined with the Community of Inquiry as the foundation for understanding the impact of storytelling.

\section{Purpose of the Study}

Normally storytelling consists of the instructor telling the story, so the students can learn from the storyline. It is a teaching tool that is commonly used in the lecture format, as the instructor demonstrates through a story the application of the concept being shared (Geres, 2016). We then asked, what if students applied the concepts of storytelling and created their own stories? Our overarching research question thus inquires: if a student learns how to tell a story and then tells a story in an online discussion, does this experience create or enhance the opportunity for construction of knowledge through a community of inquiry? This leads to the current preliminary study, endeavoring to understand from the students themselves if a guide to storytelling is beneficial in the construction of knowledge through the community of inquiry created in a class discussion (Cooper \& Striven, 2017).

The purpose of this phenomenological qualitative study was to understand the perceived differences of students who have specific directions in storytelling as well as their own perception as to storytelling enhancing their discussions overall. Along with a community of inquiry created by the students, this study explored constructing knowledge through storytelling from two points of view: formal survey questions and focus groups. By examining the concepts of those who have been prepared in storytelling and those with no formal training, we can better understand the value of storytelling as a means to construct knowledge in this environment by addressing a specific topic and the skills one needs. Discussions are often challenging in assessing knowledge gained. The goal for this research is to see if there is value to teaching how to tell a story and then asking for a story rather than just an answer to a discussion assignment.

\section{Methodology}

\section{Participants}

This study describes the common meaning of experiences of a specific phenomenon. The researchers reported data that reduced the experience of storytelling to a central meaning (Creswell, 2013; Patton, 2015). Participants were recruited from two sections of a Freshman First-Year Seminar classroom at a liberal arts university in 
the Midwest. The first section had 24 students with over $50 \%$ completing an online survey (see Appendix A). Another section of 17 students participated and took part in a focus group related to the online storytelling posts. The participants were between 18-24 years of age and primarily identified as Caucasian. There were almost equal numbers of females and males in section one ( $\mathrm{F}=13, \mathrm{M}=11)$. Participants in the second section were primarily female ( $\mathrm{F}=15, \mathrm{M}=2)$. Both sections posted their responses to each story online, while one had a robust conversation as a focus group in class rather than completing the survey. The focus group was used for the second section to help the researchers obtain more in-depth information about the storytelling experience and also to triangulate the data. The same questions were covered in both formats. All students had to complete the assignment, but not all were required to answer the questions or discuss in the focus group. Two of the researchers were instructors in the class, with a third external researcher to guide the activities. From the focus group discussion, field notes were recorded that augmented the online survey. Survey results were compiled using Survey Monkey. No identifiers of respondents or specific responses were reported. The focus group data was collected by the faculty through note taking during class. This data was then coded based on recurring themes and comments. The survey data and the focus group data were then analyzed and are reported in the results section of this paper.

\section{Procedures}

The research was conducted in a face to face classroom; the assignment was completed in the Canvas learning management system through a discussion board although students shared in the face to face classroom as well. All participants were asked to tell a story about an experience in which they were engaged at the University, such as attending a sporting event or guest speaker on campus. Students were given the story telling procedure as outlined in the next paragraph, and then asked to relate this story to one of six literacies, which included Analytical and Critical Thinking, Community and Civic Responsibility, Scientific Inquiry, Ethics and Values, Literary and Artistic Expression and Interdisciplinary and Integrative Thinking. For a more detailed explanation of the literacies, please refer to Appendix A. The outcome desired for this assignment is for the students to develop knowledge about the University's culture and purpose in education through a discussion about an activity they attended on campus or were engaged in on campus. One of the learning outcomes for the Freshmen First Year seminar course is to provide a foundational understanding of the importance of a liberal arts education. This assignment is designed to enhance this understanding and knowledge for the students through a lived experience they can share in the discussion.

Telling a story was the assignment for the student to complete. A storytelling guide was developed and shared with half of the participants (see Appendix B). The guide was based on a summary of The Art of the Nudge. In this book the authors describe the very clear steps needed for interaction of readers that can then lead to construction of knowledge (Geraci \& Miles, 2015). The main criteria for storytelling based on this book include the following summarized list which was expanded with detail in Appendix B for the students. 
Character, Scene, When, Struggle, Difficulties, who is affected

Turning Point:

Event, AH HA Moment

New Beginning:

Today, Significant difference, Outcomes, Results

Half of the students received guidance on how to tell a story, while the other half had no formal storytelling guidance. The purpose was to see if the storytelling guide assisted students as they prepared their story. Once the students had the story telling guide, they were then directed to tell their story in writing and share it in the discussion board based on their experience of the social event they attended as it applied to the literacy. Students were then asked to reply to each other in the discussion board asking clarifying questions about the personal stories written. Once the assignment was completed, students were then asked to share their thoughts about the experience of telling a story as an assignment in constructing knowledge. Questions were also asked of the students about their perception of the use of a guide or lack of use of a guide as well as their perception of using stories in the discussion.

The faculty reviewed the stories in the learning management system and noted the relation to the University literacy as well as the interaction and responses between students. In one section students were invited to participate in a survey through a letter shared with them in class once they completed the assignment. Participants were asked a series of questions about their experience after they completed the storytelling exercise (see Appendix C). The results were reported in Survey Monkey. In a second section students were asked to share their thoughts about the guide as well as the overall learning opportunity through the story telling activity. All students were given the story telling guide after the research was completed to ensure that all participants received the same classroom learning information. The survey and notes provided qualitative data that was analyzed to compare the difference in the experience for the students constructing the story in the guided storytelling and the unguided storytelling discussion assignment as well as their perception of the value of storytelling overall.

\section{Results}

\section{Triangulation}

Data was triangulated through three means: student surveys, focus groups, and literature. In the literature, Garrison et al. (2011) noted that collaboration in an educational experience creates an emergence of knowledge through self-efficacy which leads to an environment where the learning that occurred through storytelling was shared with others. This was demonstrated by student stories that were shared with others in class; this helped develop the student's learning experience. As an example, responses from our student focus groups were consistent with those on the survey. These responses also supported the literature that an emergence of knowledge can be developed through storytelling. The survey indicated that students found storytelling to be a means of creating opportunities for knowledge and understanding beyond their 
previous understanding of the topic they reported. Although a storytelling guide was offered to one group of students, others told the story without being offered the guide. In each instance, students did not have a significant feeling either for or against the guide. Most students mentioned that they felt they already knew how to tell a story, either through tacit or implicit direction prior to receiving the guide for the study. Only two students reported the guide was useful.

The focus group was engaged to compare the thoughts from the students who received the guide to those which appeared on the survey. The learning from the storytelling exercise and the creation of knowledge showed similar results, regardless of whether they had the storytelling guide as well as others who participated in the focus groups.

\section{Positive Experience}

The results from the study demonstrated that storytelling in the classroom was a positive experience and that it did enhance the level of overall classroom participation.

Question 4 in the student survey asked: Did you find you extended your storytelling of the topic of discussion in class by creating a story?

Around $70 \%$ of the responses were considered positive. One student shared, "Yes I understand now we are always having group chats which involves us with the class even if we had a different thinking....all came to an understanding." Another shared, "It was a different way to approach an activity for this class, which was fun." It appeared the interest in storytelling led to an increased level of activity, with postings by students beyond the required post and response.

The online discussion provided an opportunity for students who are not comfortable in social settings an opportunity to interact as well. When the faculty introduced the storytelling concept to students, some students were reluctant to engage in the exercise. A few students shared with the faculty their hesitation to speak in class, but the online storytelling environment allowed them the opportunity to share without having to be what they perceived as being "social."

\section{Engaging through a Community of Inquiry}

Students shared that they felt it was easier to engage with the topic being shared overall. It appeared students felt the exercise improved their understanding and recognition of a community of inquiry. Question 6 of the survey asked: Did you find you understand the topic in a new way by listening to other stories? The responses in question 7 also benefitted the notion of a Community of Inquiry. The question asked: What are your overall feelings (or thoughts) on storytelling in a discussion assignment? Students had this to say when reflecting on the Community of Inquiry: "I thought it was a cool way to interact with others. I also think it was nice to read what others thought." This creates a social interaction for the student that Lev Vygotsky (Pass, 2007) viewed as necessary to provide an environment for constructing new knowledge. Another noted: "I replied to their stories and attempted to put myself in their shoes to better understand the 
stories being told" which built the community. Students overall felt more engaged with the classroom as stories were being shared, and the faculty noticed this as students asked questions of each other in the classroom both online and in person. As one student wrote, "I think reading the other stories was good to see effective ways to tell my story." It helped students feel connected to the group. The faculty noticed a different level of discussion after the assignment. The classroom became a new "community" that was still discussing some of the stories shared, with additional inquiry through the students' own cognitive understanding in an educational setting.

The connection of the cognitive process of storytelling, along with the social aspect of sharing a story with others and reading other stories in a learning environment, created the opportunity for a positive educational experience (Garrison et al., 2010)

\section{Expanded opportunity for Constructivism}

The interaction in the classroom along with the stories shared as they related to the literacy increased the amount of activity in the storytelling assignment. Question 6 probed the student by asking: Did you find you understood the topic in a new way by listening to others' stories? The limited study does not provide enough evidence to confirm this; however, when students were asked, they did feel this was a way to construct knowledge through storytelling. Further comments included: "I could see how it impacted them and how literacies can be applied to many aspects of our lives" and "I believe that reading classmates stories provided me with a new understanding of different literacies and the people themselves." One student mentioned she was intimidated but still enjoyed the opportunity to communicate via storytelling. Another student noted he enjoyed learning by "hearing" the stories of others. As a means to make sense of the assignment, he often would reference certain aspects of his home environment to augment the learning process, demonstrating his construction of knowledge based on his understanding.

\section{Teaching Storytelling}

A large part of this study was seeking to understand if it helped to teach students how to tell stories. However, the findings and results demonstrated that this did not appear to help, nor did the students appear to feel they needed the assistance. There were a few challenges, one being that not all students responded. The majority of students shared they already knew how to tell a story: "I already knew how to tell a story" and "we learned how to tell stories in high school." One student shared, "I am already good at telling stories." Only two students shared that "I used the handout as a guide and it was helpful" and the guidance "taught me a new way of storytelling." In the focus group one student shared she was excited to tell a story as a means to share and learn with the class. Others in the focus group did not share as much enthusiasm as many felt they were proficient in storytelling. Some mentioned they learned storytelling techniques in high school while a few others thought it was somewhat common sense. The focus groups and those with specific instructions on storytelling as 
well as those with no instruction appear to agree that teaching this skill had little influence on their ability to tell a story.

\section{Conclusion}

Historically, storytelling has been a standard for communication to assist students in learning cognitively (Hamstra, 2017). Its deep roots in graphical illustration, word of mouth, written and other formats has constructed knowledge and depth of understanding for thousands of years. While storytelling is not new to higher education, this research addressed the perceived effect on students of providing specific directions on storytelling in relation to making sense of the story and constructing knowledge through a community built using the online discussion forum (Cherry, 2017; Powell \& Kalina, 2009). The fundamental research question can then be posed: Does guided storytelling enhance the community of inquiry by providing an environment for the construction of knowledge by the student in the classroom? The researchers found that the majority of students in the study felt they already had an understanding of the components of storytelling, so teaching them how to tell a story was not valuable in their minds. However, they did appreciate the guidance on how to complete the assignment, and the overall experience of telling a story was a positive experience. Taking this same process and replicating it with additional assignments would provide an opportunity to expand on the educational value of the story telling added to other disciplines. It was evident that this discussion enhanced the level of engagement on the assignment, building a community of social interaction in an educational setting.

Those who were not given directions on how to tell a story noted that they used previous experiences as a guideline. They either used learning from past experiences or just said what came to mind. While members of each group told stories based on their understanding of the components and principles of storytelling, each respondent felt storytelling generated a significant new understanding of the topic. Those who responded to the survey shared a positive, enhanced learning experience.

In the community of inquiry model, there are three pieces: cognitive, social and teaching, which culminate in the educational experience. In the constructivism model there are social learning tasks, and personal understandings, similar to the community of inquiry. The storytelling discussion board assignment is used to provide an environment for knowledge to be constructed through the educational experience. Students created a social environment by telling stories in the online discussion board. From there the learning can occur by constructing new knowledge based on their own cognition. While this is a limited study, it does appear these three areas are important as students socially interact, creating a community where new knowledge can be constructed based on the stories shared. 
Figure 3

New Model Created Out of the Combined Community of Inquiry and Constructivism Model

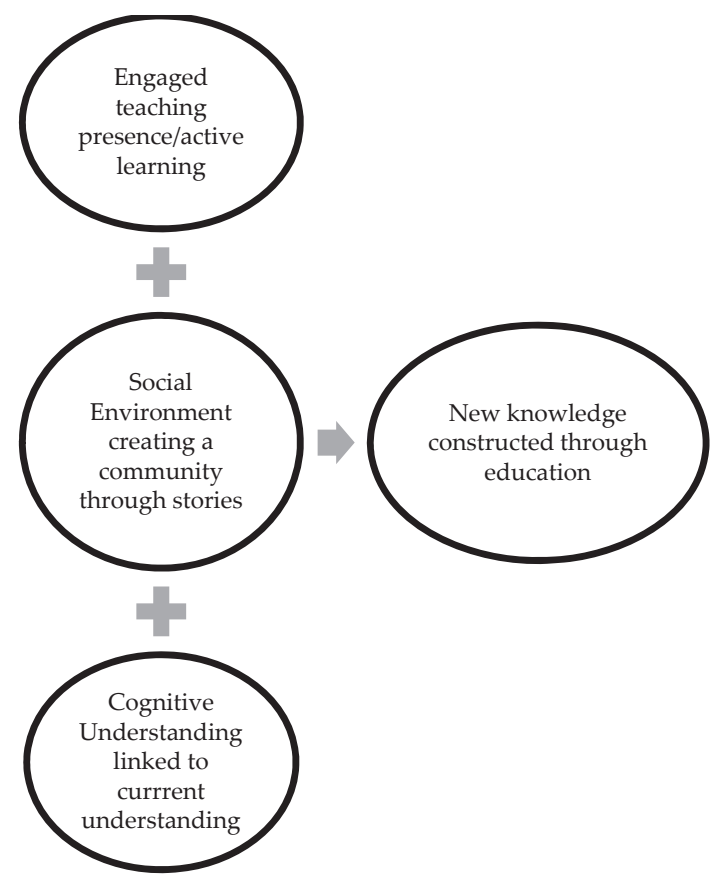

\section{Further Research}

Further research on using storytelling in online discussion boards would help to confirm that the construction of knowledge does exist through the development of a social community. Additionally, new research may focus on whether university students continue to construct knowledge through storytelling as they move closer to graduation and if this becomes a valuable tool in the work place. There is also an opportunity to explore the use of storytelling as a tool for students to explain complex issues or to encourage a community of inquiry when working on group projects, sensemaking exercises, or presenting a unique or new idea. Additional research could also add value to see if online versus face-to-face applications of storytelling would be different. One student shared how storytelling created a "safe space" which could be further researched. The current study shows that the use of storytelling did create a positive learning opportunity overall from the perception of the students. 


\section{References}

Brooks, M. G., \& Brooks, J. G. (1999). The courage to be constructivist. Educational Leadership, 57(3), 18-24.

Cherry, W. R., Jr. (2017). The importance of story and storytelling in the classroom. Knowledge Quest, 46(2), 50-55.

Chow, H. M., Mar, R. A., Xu, Y., Liu, S., Wagage, S., \& Braun, A. R. (2014).

Embodied comprehension of stories: Interactions between language regions and modality-specific neural systems. Journal of Cognitive Neuroscience, 26(2), 279-295. doi: 10.1162/jocn_a_00487

Cooper, T., \& Scriven, R. (2017). Communities of inquiry in curriculum approach to online learning: Strengths and limitations in context. Australasian Journal of Educational Technology, 33(4), 2237. https://doi.org/10.14742/ajet.3026

Creswell, J. W. (2013). Qualitative inquiry \& research design: Choosing among five approaches ( $3^{\text {rd }}$ ed.). Sage.

Dunlap, J., \& Grabinger, R. (1996). Rich environments for active learning in the higher education classroom. In B. G. Wilson (Ed.), Constructivist learning environments: Case studies instructional design (pp. 65-82). Educational Technology Publications.

Fahy, P. (2007). The occurrence and character of stories and sorytelling in a computer conference. Distance Education, 28(1), 45-63. doi: 10.1080/01587910701305301
Garrison, D. R. (2009). Implications of online learning for the conceptual development and practice of distance education. Journal of Distance Education, 23(2), 93-104.

Garrison, D. R., Cleveland-Innes, M., \& Fung, T. S. (2010). Exploring causal relationships among teaching, cognitive and social presence: Student perceptions of the community of inquiry framework. Internet in Higher Education, 13, 31-36. https://doi.org/10.1016/j.iheduc.2009.10.0 02

Garrison, D. R., \& Vaughan, N. D. (2008). Blended learning in higher education: Framework, principles, and guidelines. Jossey-Bass.

Geraci, J., \& Miles, C. (2015). The Art of the Nudge. Aviva.

Geres, K. (2016). Resilience through storytelling in the EAL classroom. TESL Canada Journal, 62. http://doi.org/1018806/tesl.v33i0.1245

Hamstra, C. (2017). Complexity storytelling: The science of complexity within the art of communication. Emergence: Complexity \& Organization, 1-6. https://doi.org/10.emerg/10.17357.076ac03 18796a9ab519c0a5463154e88

Keoning, J. M., \& Zorn, C. R. (2002). Using storytelling as an approach to teaching and learning with diverse students. Journal of Nursing Education, 41(9), 393-9. 
Krahenbuhl, K. S. (2016). Studentcentered education and constructivism: challenges, concerns, and clarity for teachers. The Clearing House: A Journal of Educational Strategies, Issues and Ideas, 89 (3), 97-105.

Mar, R. \& Oatley, K. (2008). The function of fiction is the abstraction and simulation of social experience. Perspectives on Psychological Science, 3(3), 173-192. https://doi.org/10.1111/j.17456924.2008.00073.x

Pass, S. (2007). When constructivist Jean Piaget and Lev Vygotsky were pedagogical collaborators: A viewpoint from a study of their communications. Journal of Constructivist Psychology, 20(3), 277-282.

https://doi.org/10.1080/1072053070134794 4

Patton, M. Q. (2015). Qualitative research $\mathcal{E}$ evaluation methods: Integrating theory and practice ( $4^{\text {th }}$ ed.). Sage.

Phillips, C., Bassell, K., \& Fillmore, R. (2017). Storytelling and reflective pedagogy: Transforming nursing education through faculty development. American Journal of Health Sciences 8(1), 112.

https://doi.org/10.19030/ajhs.v8i1.9953
Powell, K. C., \& Kalina, C. J. (2009). Cognitive and social constructivism: Developoing tools for an effective classroom. Education, 130(2), 241-250.

https://search.ebscohost.com/login.aspx? direct=true\&AuthType $=\mathrm{sso} \& \mathrm{db}=\mathrm{f} 5 \mathrm{~h} \& \mathrm{AN}$ $=47349084 \&$ site $=$ eds-live

Sterman, J. D. (1991). A skeptic's guide to computer models. In G. O. Barney (Ed.), Managing the nation: The microcomputer software catalog (pp. 201-229). Westview Press.

van Rensburg, G. H., Botma, Y., Heyns, T., \& Coetzee, I. M. (2018). Creative strategies to support student learning through reflection. South African Journal of Higher Education, 32(6), 604-618.

https://doi.org/10.20853/32-6-2888

Zak, P. J. (2015). Why inspiring stories make us react: The neuroscience of narrative. Cerebrum: The Dana Forum on Brain Science, 2.

https://www.ncbi.nlm.nih.gov/pmc/articl es/PMC4445577/ 


\section{Appendices}

\section{Appendix A}

Analytical and Critical Thinking

The student with critical thinking literacy will be able to gather, evaluate, and communicate information effectively; meet the basic computing demands of contemporary life; recognize varieties of problem-solving strategies; and be able to contribute to desirable changes or help preserve and transmit knowledge for the good of society.

1.1 Demonstrate mastery of the basic skills in communication, technology, and information management. 1.2 Recognize diversity in the processes and methods of critical thinking and problem-solving. 1.3 Differentiate the variety of approaches for examining and using information, and their technological applications in contemporary life. 1.4 Utilize tools for gathering, retrieving, evaluating, and communicating information and data for various purposes. These tools should include the basic skills in writing, speaking, listening, computing, and problem solving. 1.5 Synthesize knowledge gathered from different cultures in communication and problem-solving efforts.

\section{Community and Civic Responsibility}

The student with community and civic literacy will be able to see the complexity of social, political, and economic systems and problems on the national and international scene, and then develop ways that would contribute to the solution of such problems through effective citizenship participation.

2.1 Demonstrate understanding of the workings of American social, political, and economic systems. 2.2 Recognize the existence of diverse alternative systems and their necessary global relationships. 2.3 Trace the geographical and historical roots which are shaping these systems. 2.4 Analyze the tools for responsible citizenship involvement and for participation in economic and social endeavors. 2.5 Describe the diverse values, beliefs, ideas, and worldviews found globally into personal community and civic activities.

\section{Scientific Inquiry}

The student with scientific literacy will demonstrate an appreciation of science as a means of acquiring knowledge; scientific knowledge being the cumulative result of applying logic to sensory data for the purpose of developing theories that explain natural phenomena.

3.1 Apply appropriate science concepts, principles, laws, and theories to interact with the universe. 3.2 Utilize the processes of science in solving problems, making decisions, and furthering one's own knowledge of the universe. 3.3 Demonstrate the ability to make informed personal decisions about issues that involve science (i.e., health, the sustainable use of resources, etc.) in ways that are consistent with the values that underlie science. 3.4 Demonstrate numerous manipulative skills associated with science and technology (i.e., measurement, data collection, and interpretation of results). 3.5 Analyze and critically respond to the ways that science is represented in 
other disciplines, in popular culture, and in the media. 3.6 Demonstrate understanding of the multicultural history and experimental nature of scientific knowledge.

\section{Ethics and Values}

The student with ethical literacy is sensitive to questions about ethics and values, appreciatively and critically aware of differing systems of ethics and values, in possession of tools for analyzing questions about ethics and values, and engaged in the process of putting these things together into a consistent set of personal and socially responsible values that one feels comfortable upholding and perhaps revising throughout life.

4.1 Discuss the importance of ethics and values in human life, how both interact with attempts to objectively and subjectively understand the world. 4.2 Identify and deploy methods for analyzing and resolving disputes about ethics and values. 4.3 Recognize the diversity and similarities in value systems held by different cultures and cocultures. 4.4 Demonstrate the ability to seek common goals and principles through which different cultures can work together to solve common problems. 4.5 Develop a personal and socially responsible system of ethics and values that one feels comfortable revisiting and perhaps revising throughout one's life.

\section{Literary and Artistic Expression}

The student with literary and artistic expression is one who is acquainted with various aesthetic human experiences and expressions in their historical and cultural contexts, is able to enjoy them with informed judgments, and also able to contribute to such experiences and expressions.

5.1 Identify the great themes and metaphors of human experience in various forms of aesthetic expressions to enhance the understanding of life. 5.2 Discuss diversities in the visual, verbal, and performing arts and the origins and reconciliation of such diversities. 5.3 Compare and contrast the role of various art forms from a range of societies as both records and shapers of language and cultures. 5.4 Engage in perceptive and open-minded enjoyment of aesthetic experiences. 5.5 Create aesthetic works that reflect awareness of self and social contexts.

\section{Interdisciplinary and Integrative Thinking}

The student with interdisciplinary and integrative literacy possesses the ability to make connections across courses and connect coursework to his/her academic, professional, and civic lives. The student will be able to consider problems from several different perspectives and develop and test his/her holistic understanding of an issue, evaluate how various disciplines would conceive of solutions, and relate his/her learning to issues outside of academia.

6.1 Compare differences and similarities among the disciplines in terms of central concerns, values, methodologies, and relationship to public life. 6.2 Synthesize diverse perspectives to achieve an interdisciplinary understanding. 6.3 Discuss the relationships among academic knowledge, professional work, and the responsibilities of local and global citizenship. 6.4 Utilize multiple modes of inquiry, expression, and processes for decision-making. 


\title{
Appendix B
}

\section{Storytelling Condition:}

Please share in the discussion board information about an activity you were engaged in at the University, apply at least one of the literacies and discuss how this impacted you as a person and a student at the University by telling a story in the discussion posting. This portion will be worth 20 points. Then read other student's postings and reply to at least two other students for an additional 20 points, for a total of 40 points.

\section{DO NOT SHARE THE FOLLOWING WITH OTHERS:}

Please review below how to tell a story:

How to Tell a Story:

Has a beginning, a middle and an end with a character who changes by the conclusion. Please follow the outline below to share your story about your engagement at this University.

\author{
Beginning \\ Character \\ Scene \\ When \\ Struggle \\ Difficulties \\ Who is affected \\ Turning Point \\ Event \\ AH HA Moment \\ New Beginning \\ Today \\ Significant difference \\ Outcomes \\ Results \\ CI Squared LLC (2015) \\ Control Condition:
}

Please share in the discussion board information about an activity you were engaged in at the University, apply at least one of the literacies and discuss how this impacted you as a person and a student at the University by telling a story in the discussion posting. This portion will be worth 20 points. Then read other student's postings and reply to at least two other students for an additional 20 points, for a total of 40 points. 


\section{Appendix C}

Did you receive any guidance or information from your faculty or classmate on how to tell a story? Please explain.

If yes, did you use the guidance and was it helpful? Please explain and give feedback or suggestions on the guidance received.

If no, how did you develop your story to tell? Please explain.

Did you find you extended your understanding of the topic of discussion in class by creating a story? Please explain.

Did you participate in the discussion when classmates told their story?

Did you find you understood the topic in a new way by listening to other stories?

What are your overall feelings (or thoughts) on storytelling in a discussion assignment?

Donna M. Ehrlich, PhD, is Associate Professor of Computer Information Management, Park University and holds a PhD from the Graduate School of Computer and Information Sciences, Nova Southeastern University. Dr. Ehrlich spent 15 years in Fortune 500 companies in technology application and leadership before becoming a full time faculty in higher education. Dr. Ehrlich's dissertation research explored the adaptation of system processes with technology applications, she continues this research with interests in classroom improvements adapting the educational experience for the student. Dr. Ehrlich's research interests also include Autonomous Vehicles and Smart Cities using Edge Computing in the analysis of data.

Dr. Jeff Ehrlich is an Associate Professor in the College of Management at Park University. Prior to returning to faculty, he served as the Executive Director of Park University in Gilbert, Arizona. Starting with Park University in 2011, Ehrlich has served several roles including system wide University Interim President, Associate Dean, Director of Strategic Initiatives, and University Faculty Senate President for two consecutive terms. He was awarded tenure in 2017. He has held numerous roles in other academic institutions in his 19 years as a faculty and administration. Ehrlich completed an EdD in Healthcare Leadership from the College of Saint Mary in Omaha, Nebraska. Ehrlich also attended Oklahoma State University where he was awarded the Maxine and Robert Kamm Distinguished Graduate Endowed Fellowship. He completed a master's degree in Business Administration in 1997. He was awarded the Park Family Award presented by Park Student Government Association, the Department of Athletics Donald Breckon Lifetime Achievement Award, and the Kansas City Northland and Regional Chamber of Commerce Excellence in Higher Education Award.

April Haberyan, PhD, RN, is the Nurse Residency Program Manager at Truman Medical Centers in Kansas City, Missouri and holds a PhD in Social Psychology from the University of Nebraska- Lincoln and a Master's in Adult Psychiatric Mental Health Nursing from the University of Rochester. As a former faculty member at Park University she taught Abnormal Psychology, Social Psychology and Disaster Psychology. She received the Missouri Governor's Excellence in Education award in 2010. Her research interests include suicide prevention, disaster response and self-regulated learning. During her free time, she is a consultant, trainer, and responder for Disaster Management International. 